\section{Anatomical variants in the floor of the third ventricle; implications for endoscopic third ventriculostomy}

\author{
Nobuhito Morota, Takeya Watabe, Takashi Inukai, Kazuhiro Hongo, Hiroshi Nakagawa
}

landmarks that require special attention during third ventriculostomy.

(f Neurol Neurosurg Psychiatry 2000;69:531-534)

Keywords: hydrocephalus; endoscopy; third ventriculostomy

Endoscopic third ventriculostomy plays an important part in the treatment of hydrocephalus. ${ }^{1}$ Anatomical landmarks are well established and the procedure is safe in well experienced hands. Recently we have encountered two adult patients who were diagnosed as "long standing overt hydrocephalus in the adult (LOVA)"2 and in whom the floor of the third ventricule was seen to be completely different from the normal anatomy. We report the cases and surgical techniques, and discuss the relation between hydrocephalus, empty sella, and persistence of infundibular recess which were associated with the patients.

\section{Case report}

CASE 1

A 31 year old woman had had chronic headaches, which increased in frequency and were accompanied by dizziness. Brain CT showed marked ventriculogmegaly with flat-
Department of Neurological Surgery, Aichi Medical University, Nagakute-cho, Aichi-gun, Aichi 480-1195, Japan N Morota

T Watabe

T Inukai

K Hongo

H Nakagawa

Correspondence to: Dr N Morota nobu@masa.go.jp

Received 18 February 2000 and in revised form 5 April 2000

Accepted 12 April 2000

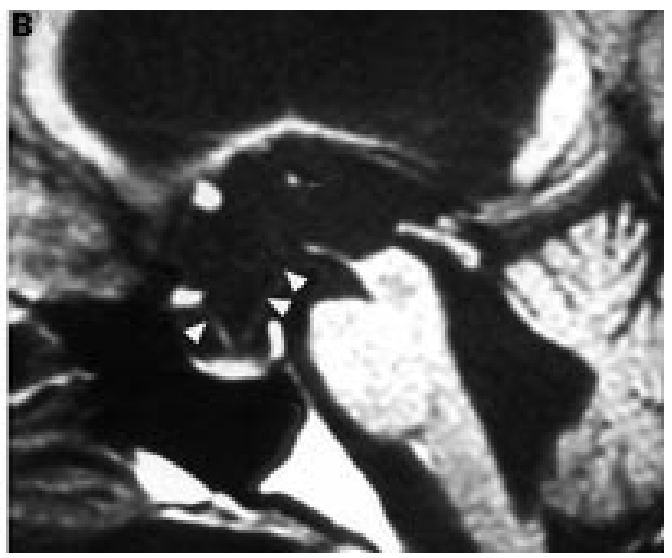

Figure 1 (A) Preoperative MRI (T1 weighted image, sagittal view) of case 1 showed marked enlargement of the third and fourth ventricles. The anterior part of the floor of the third ventricle herniated into the empty sella (large arrow head). The infundibular recess was completely dilated. Note that the rest of the anterior part of the floor of the third ventricle (between the dorsum sellae and mamillary body) was hanging down over the basilar artery and pons (small arrow heads). (B) Postoperative MRI taken 2 weeks after surgery showed no ballooning of the floor of the third ventricle. The shape of the anterior part of the floor of the third ventricle became concave after endoscopic third ventriculostomy (small arrow heads). 


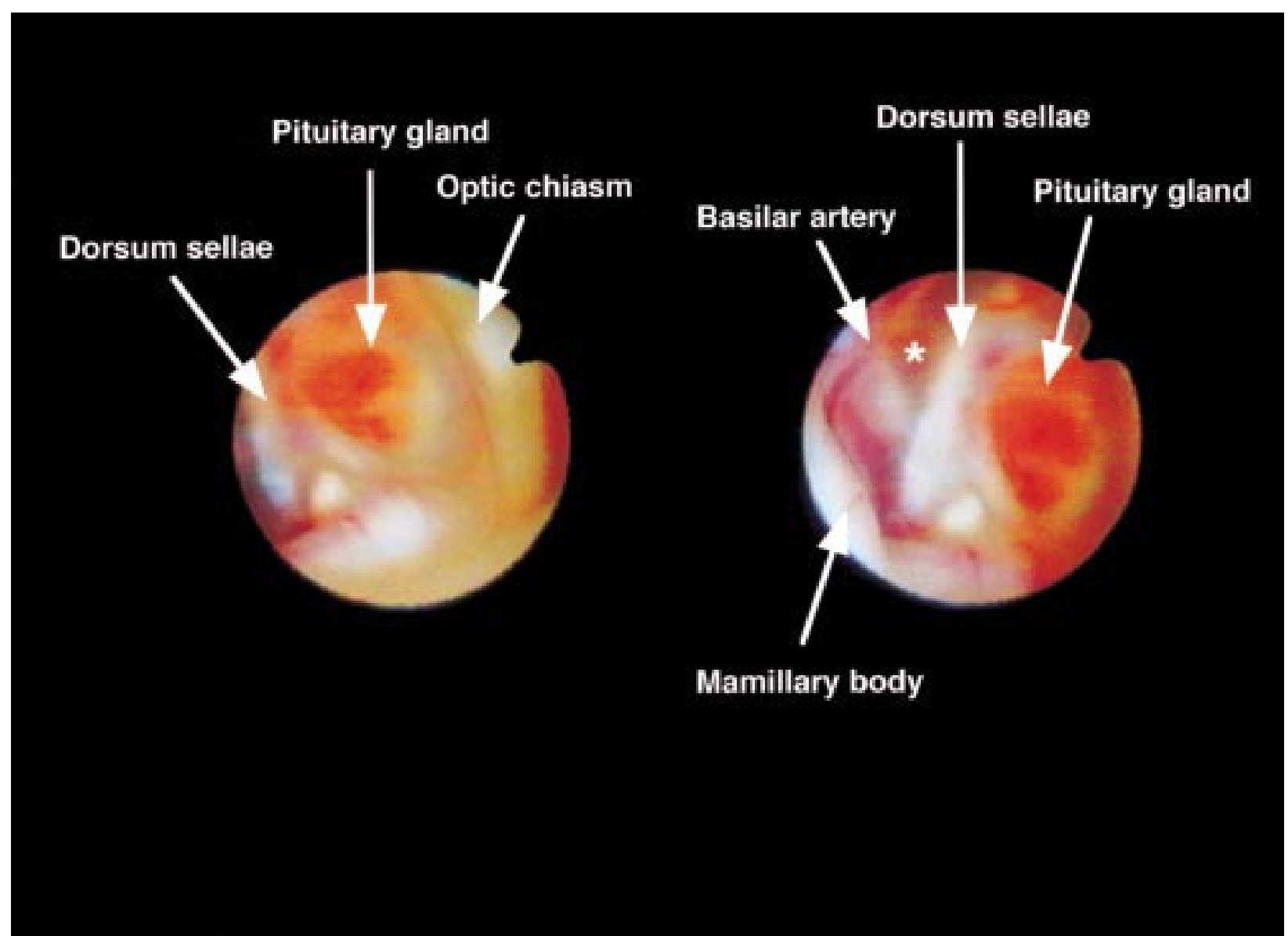

Figure 2 Intraoperative photographs of the floor of the third ventricle in case 1 . The pituitary gland coloured orange was directly in the bottom of the pituitary fossa between the optic chiasm and dorsum sellae. The basilar artery was directly visible through the floor of the third ventricle which was hanging down on it. The asterisk indicates the point where third ventriculostomy was made.

tening of the cortical sulci. She was intelligent and had no neurological deficit. The optic fundi were normal in appearance. Neuroradiologically, all ventricles and the aqueduct were enlarged suggesting obstruction of fourth ventricular CSF outflow. The floor of the third ventricle was bowed inferiorly, hanging down ventral to the pons as a part of the dilated infundibular recess which continued into the empty sella. A CT cisternogram showed normal CSF circulation and no delay in absorption of contrast material (fig 1). Based on the symptoms and radiological findings, she was diagnosed as having obstructive hydrocephalus with LOVA. During endoscopic third ventriculostomy, it was noticed that the normal anatomy of the floor of the third ventricle was lost. Instead of the tuber cinerum and infundibular recess, there was a reddish spot in the centre, which turned out to be the remnant of the pituitary gland at the bottom of the sellar turcica. Behind the dorsum sellae, the basilar, posterior cerebral, and superior cerebellar arteries could be seen through the herniated thin wall of the third ventricle. Third ventriculostomy (fig 2) was placed on the dorsal wall of the herniated infundibular recess. Using biopsy forceps, a small hole was bluntly made and was dilated by an $2 \mathrm{Fr}$ Forgaty balloon catheter.

Her symptoms disappeared within a week after surgery. Brain MRI showed that the floor of the third ventricle changed to concave from the bulged shape seen preoperatively. The ventricular size gradually decreased in the next 6 months (fig 3).

\section{CASE 2}

A 35 year old woman with neurofibromatosis type 1 underwent posterior fossa decompression for Chiari 1 malformation when aged 28. Marked ventriculomegaly with associated intractable chronic headache persisted despite surgery. She had no neurological deficit and the optic fundi were normal in appearance. MRI showed a panventricular enlargement. In the sagittal view, the herniated cerebellar tonsil sagged into the dorsal cervical canal with no CSF flow on the phase contrast cine MRI. It was again noticed that the floor of the third ventricle was hanging down ventral to the pons, forming a dilated infundibular recess which herniated into the partially empty sella. During endoscopic third ventriculosotomy the appearance of the floor of the third ventricle was almost similar to that of case 1 and third ventriculostomy was carried out in an identical fashion.

Postoperatively her symptoms improved within a week. The postoperative MRI showed the same change in the shape of the floor of the third ventricle as in case 1 . 


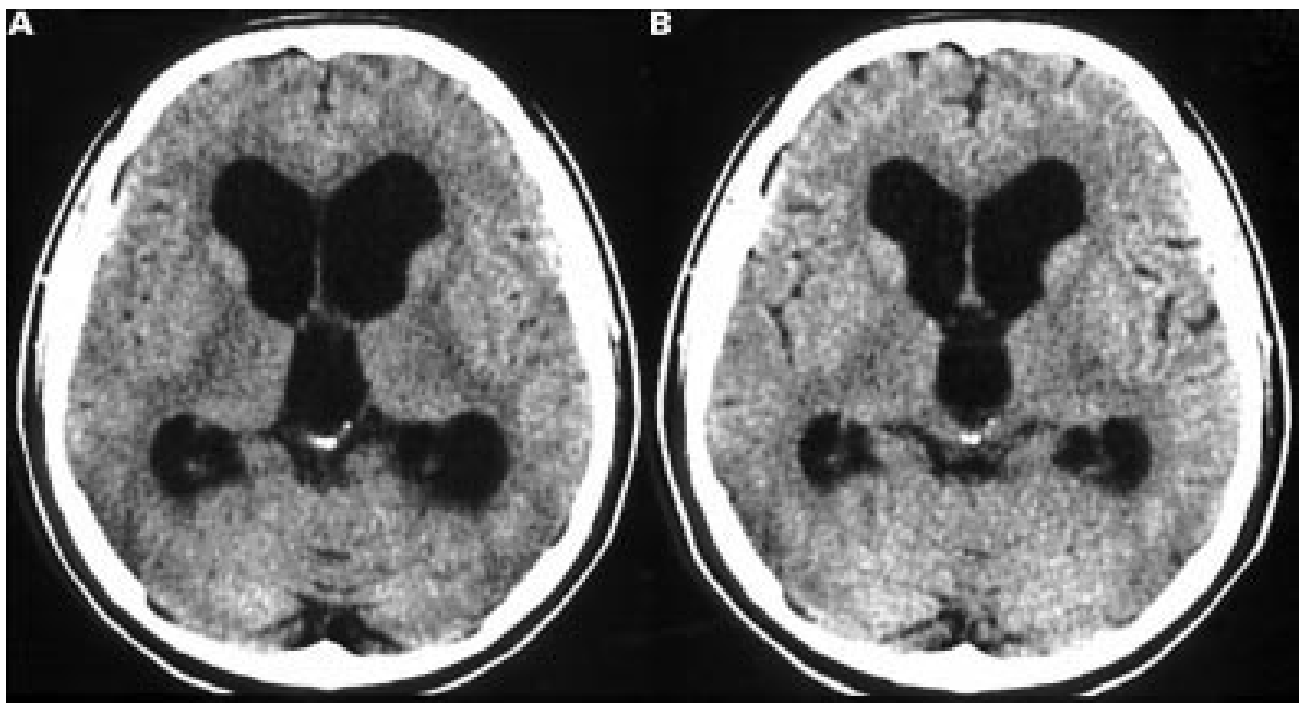

Figure 3 (A) Preoperative CT disclosed marked dilatation of the lateral ventricle. Note that the brain cortex was tight and opacification of the cortical sulci was poor. (B) Postoperative CT taken 2 months after surgery showed mild reduction in ventricular size. The cortical sulci were more visible because of the enlarged subarachnoid space after surgery.

\section{Discussion}

Endoscopic third ventriculostomy is a relatively straightforward procedure unless there are anatomical variations present on the floor of the third ventricle. ${ }^{1}$ In the cases of LOVA we have experienced the preoperative MRI provided us with important information on the anatomy of the floor of the third ventricle. The infundibular recess was dilated and the anterior inferior floor of the third ventricle was hanging down ventral to the pons and herniated into the empty sella. The dorsal half of the herniated floor was positioned overriding the basilar artery trunk. These findings are compatible with those found during the endoscopic surgery.

Endoscopic third ventriculostomy is technically demanding in patients with abnormal anatomy of the floor of the third ventricle. In our cases standard ventriculostomy on the tuber cinerum was impossible as the floor herniated into the empty sella. The site which we chose to perform the ventriculostomy was the floor which was hanging ventral to the pons. Ventriculostomy was made at the left corner surrounded by the dorsum sellae, the basilar artery trunk, and the superior cerebellar artery. It was selected because it was easy to see the area using a flexible endoscope without excessive flexion. It was also easy to access the left side of the floor when the endoscope was introduced through the right foramen of Monro. Dynamic pulsation of the floor wall occurred after blunt penetration and balloon dilatation ventriculostomy. Postoperative MRI disclosed a concave shape in the floor of the third ventricle which had shown ballooning preoperatively.

The presence and development of hydrocephalus, empty sella, and persistence of an infundibular recess seem to be interrelated. An empty sella is three times more common in patients with communicating hydrocephalus than normal subjects. ${ }^{3}$ Additionally raised ICP and obstructive hydrocephalus may also cause empty sella syndrome. ${ }^{4-7}$ The shape of the anterior part of the third ventricle is also influ- enced by the intraventricular pressure. ${ }^{8}$ The anterior part (the premammillary portion) of the floor of the third ventricle consists of a very thin layer of grey matter of the hypothalamus and can protrude into the sella under high intraventricular pressure. ${ }^{9}$ The likelihood of developing this anatomical change is higher with the presence of a pressure gradient between the third ventricle and the subarachnoid space, as occurs in cases of obstructive hydrocephalus. Increased intraventricular pressure also seems to play an important part in developing persistence of the infundibular recess, in a condition that is invariably associated with hydrocephalus and empty sella. $^{51011}$ None the less the association of hydrocephalus, empty sella, and the persistence of the infidbular recess is very rare. Probably other factors such as position of the optic chiasm $^{9}$ and the duration of hydrocephalus may contribute to the association of the anomalies. It is interesting that our cases were young women with LOVA and most of the other cases are elderly patients; there are no reported cases in children of hydrocephalus, empty sella, and persistence of the infundibular recess.

Endoscopic third ventriculostomy is a simple and safe treatment for hydrocephalus but a hidden pitfall may occur in cases where the anatomical landmarks on the floor of the third ventricle are not discernible. Loss of normal anatomical landmarks will lead to a technically demanding third ventriculostomy. Attention should be paid to the preoperative MRI evaluation of the shape of the floor of the third ventricle, especially in the case of longstanding hydrocephalus.

1 Jones RFC, Steining WA, Brydon M. Endoscopic third ventriculostomy. Neurosurgery 1990;26:86-92.

2 Oi S, Sato O, Matsumoto S. Neurological and medicosocial problems of spina bifida patients in adolescence and adulthood. Childs Nerv Syst 1996;12:181-7.

3 Brismar K, Bajraktari X, Goulatia R, et al. The empty sella syndrome-intrasellar cisternal herniation-in normal patients and in patients with communicating hydrocephalus and intracranial tumors. Neuroradiology 1978;1:35-43. 
4 Bjerre P, Lindholm J. Adult hydrocephalus and the empty sella. Acta Neurol Scand 1984;70:201-3.

5 Kuhne D, Schwartz RG. Persisting intrapituitary recessus infundibuli. Neuroradiology 1975;10:177-8.

6 Vallee B, Besson G, Person H, et al. Persisting recessus infundibuli and empty sella: case report. $\mathcal{F}$ Neurosurg 1982 57:410-12.

7 Kulali A, Baykut L, von Wild K. Relationship between chronic raised intracranial pressure and empty sella presenting hormonal disturbances. Neurol Res 1990;12:99102 .
8 Huber P, Rivoir R. The influence of intraventricular pressure on the size and shape of the anterior part of the Cortogy 1973;5:33-6.

9 Corrales M, Trorreralba G. The third ventricle. Normal anatomy and changes in some pathological conditions. Neuroradiology 1976;11:271-7.

10 Cabanes J. Asymptomatic persistence of infundibular recessus: case report. F Neurosurg 1978;49:769-72

1 Schumacher B, Gilsbach J. A new variety of empty sella with cystic intrasellar dilatation of the recessus infundibuli. $\mathrm{BrF}$ Radiol 1979;52:862-4. 